\title{
Diurnal differences in the transcriptome of peripheral blood mononuclear cells in response to a meal
}

\author{
Rochelle Davis, Chiara Murgia, Aimee Dordevic, Maxine Bonham and Catherine Huggins \\ Department of Nutrition, Dietetics and Food, Monash University, Melbourne, Australia
}

\begin{abstract}
Eating at night has been linked to impaired glucose metabolism and dyslipidaemia that is likely a consequence of an underlying disrupted circadian rhythm in metabolic processes. An understanding of the mechanisms causing metabolic disruption after eating at night is important for prevention and management of disease risk factors. The aim of this study was to explore the transcriptomic differences in nutrient metabolism after eating a meal at night compared with the same meal in the morning. In a cross over design, 10 healthy adults fasted for $>10$ hours and then completed two acute meal challenges at $8 \mathrm{am}$ and 8pm on non-consecutive days separated by a wash out. Fasting and postprandial blood samples were collected to assess glucose and insulin responses. For a subset of five participants RNA sequencing was completed on the Illumina NextSeq500. Total RNA was extracted from peripheral blood mononuclear cells at fasting (baseline) and $2 \mathrm{~h}$ after the test meal, the quality of all samples was above RIN 8 (AATI Fragment Analyzer). Differential expression analysis was completed using the DESeq2 package. False discovery rate correction was applied at the pathway analysis level, conducted in PathVisio. Postprandial blood glucose was significantly higher at $8 \mathrm{pm}$ vs. $8 \mathrm{am}$ (208.8 $(154.1) \mathrm{mmol} / \mathrm{L} .3 \mathrm{hr}$ vs $36.4(99.6) \mathrm{mmol} / \mathrm{L} .3 \mathrm{hr}, \mathrm{p}=0.005)$ no concurrent changes in insulin responses were observed $(\mathrm{p}=0.100)$. Under fasting conditions, 704 genes were differentially expressed between morning and night, with $60 \%$ of these genes being down regulated at night. The meal challenges were associated with changes in gene expression compared with fasting in the morning 552 genes were differentially expressed and 532 genes were differentially expressed in the evening, however only $7 \%$ were commonly differentially regulated at both times of day. Pathway analysis of the differentially expressed genes identified that more immune system and signal transduction pathways were enriched after eating at night compared with morning, where as a greater number of pathways involved in lipid metabolism were enriched in the morning. The time of day a meal is consumed has an effect on which genes are differentially regulated in the acute postprandial period, and the biological pathways they are involved in. Investigating the differences in the transcriptomic response to food at night provides a greater understanding of the mechanisms underlying the phenotypic dissimilarities observed in circulating metabolic biomarkers according to the time of day.
\end{abstract}

\section{Conflict of Interest}

There is no conflict of interest 